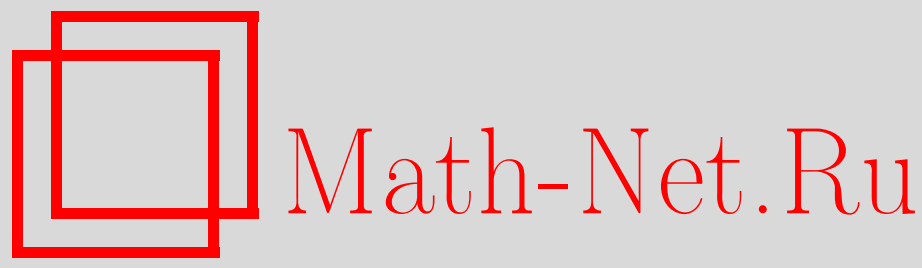

А. Кумар, Эволюционное уравнение для импульсов длительностью несколько циклов или менее чем один цикл в среде с кубичной нелинейностью, ТМФ, 2009, том 160, номер 1, 112-121

DOI: https://doi.org/10.4213/tmf6383

Использование Общероссийского математического портала Math-Net.Ru подразумевает, что вы прочитали и согласны с пользовательским соглашением http: //www . mathnet.ru/rus/agreement

Параметры загрузки:

IP : 3.81 .55 .215

26 апреля 2023 г., 16:57:38

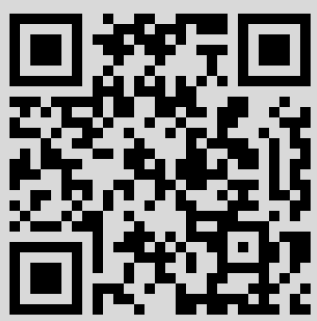




\title{
ЭВОЛЮЦИОННОЕ УРАВНЕНИЕ ДЛЯ ИМПУЛЬСОВ ДЛИТЕЛЬНОСТЬЮ НЕСКОЛЬКО ЦИКЛОВ ИЛИ МЕНЕЕ ЧЕМ ОДИН ЦИКЛ В СРЕДЕ С КУБИЧНОЙ НЕЛИНЕЙНОСТЬЮ
}

\begin{abstract}
Из нелинейного волнового уравнения для среды с кубичной нелинейностью выведено эволюционное уравнение для импульсов длительностью несколько циклов или менее чем один полный цикл. Для вещественного электрического поля получено модельное уравнение, которое использовано для анализа характеристик распространения в одномерном пространстве импульсов длительностью один цикл с длиной волны 0.8 мкм.
\end{abstract}

Ключевые слова: эволюционное уравнение для импульсов длительностью один цикл, керровская среда, нелинейная оптика.

\section{1. ВВЕДЕНИЕ}

Нескольким группам исследователей удалось успешно генерировать и стабилизировать ультракороткие импульсы, которые затем нашли свое применение в разнообразных областях физики и техники. Некоторыми авторами подчеркивалось, что нелинейное уравнение Шредингера (НУШ) непригодно для исследования характеристик распространения таких импульсов в случае, когда длительность импульса составляет один цикл и, тем более, менее чем один полный цикл. Это послужило мотивацией для поиска новых модельных уравнений, которые могут точнее описывать динамику ультракоротких импульсов в линейных и нелинейных средах [1]-[5]. Было выполнено несколько работ, авторы которых или переопределяли саму концепцию огибающей для столь коротких импульсов, или рассматривали отдельные аппроксимации для функции восприимчивости. Например, в работе [1] получено обобщенное НУШ, включающее дисперсию третьего порядка и самопроизвольное увеличение крутизны (self-steepening), а также рамановский член. В работе [2] использовалась концепция огибающей для импульсов длительностью один или несколько циклов в терминах инвариантности центральной частоты импульса при сдвиге фазы электрического поля. Исходя из этого исследователи получили эволюционное уравнение

${ }^{*}$ Department of Physics, Indian Institute of Technology, New Delhi, India. E-mail: ajitk@physics.iitd.ac.in 
для нелинейных импульсов, представляющее собой обобщение НУШ. Затем в работе [3] было получено уравнение коротких импульсов, выведенное путем аппроксимации функции восприимчивости $\chi(\omega)$ в интервале частот импульса вдали от частоты материального резонанса и много бо́льших ее. Недавно в работе [5] было выведено параксиальное эволюционное уравнение для импульсов длительностью несколько циклов или менее одного полного цикла в линейной среде без использования концепции огибающей импульса. В данной работе мы обобщим это уравнение для вещественного электрического поля на случай среды с кубичной нелинейностью, не предполагая никаких ограничений на функцию восприимчивости. Это эволюционное уравнение будет затем использовано для изучения характеристик распространения импульсов длительностью один цикл с длиной волны 0.8 мкм.

Для диспергирующей и нелинейной среды с кубичной нелинейностью уравнения Максвелла приводят к нелинейному волновому уравнению

$$
\nabla^{2} \mathbf{E}-\frac{1}{c^{2}} \frac{\partial^{2}}{\partial t^{2}} \int_{0}^{\infty} \varepsilon\left(t^{\prime}\right) \mathbf{E}\left(t-t^{\prime}\right) d t^{\prime}=\frac{\chi_{3}}{c^{2}} \frac{\partial^{2}}{\partial t^{2}}((\mathbf{E}(t) \cdot \mathbf{E}(t)) E(t)),
$$

где $\mathbf{E}=\mathbf{E}(\mathbf{r}, t)$ - вектор электрического поля импульса, $\varepsilon$ - линейная проницаемость среды, $c$ - скорость света в вакууме, $\chi_{3}$ - нелинейная восприимчивость третьего порядка в среде.

Поскольку концепция огибающей перестает работать для импульсов длительностью менее одного полного цикла, соответствующие характерные масштабы времени и длины, связанные с несущей частотой и несущим волновым вектором, отсутствуют. Поэтому мы сделаем волновое уравнение безразмерным, вводя величины $\bar{r}=\mathbf{r} / l_{0}, \bar{t}=t / t_{0}, \bar{E}=\mathbf{E} / E_{0}$, где $l_{0}, t_{0}$ и $E_{0}$ - константы, имеющие размерность длины, времени и электрического поля соответственно. Конкретные физические характеристики среды, соответствующие этим параметрам, будут выбраны ниже, когда мы представим результаты численного анализа для импульсов длительностью один цикл. В результате получаем волновое уравнение

$$
\bar{\nabla}^{2} \bar{E}(\bar{r}, \bar{t})=\frac{1}{\bar{c}^{2}} \frac{\partial^{2}}{\partial \bar{t}^{2}} \int_{0}^{\infty} \bar{\varepsilon}\left(\bar{t}^{\prime}\right) \bar{E}\left(\bar{r}, \bar{t}^{\prime}\right) d \bar{t}^{\prime}+\frac{\bar{\chi}_{3}}{\bar{c}^{2}} \frac{\partial^{2}}{\partial \bar{t}^{2}}([\bar{E}(\bar{r}, \bar{t}) \cdot \bar{E}(\bar{r}, \bar{t})] \bar{E}(\bar{r}, \bar{t})),
$$

где

$$
\begin{aligned}
\bar{c} & =\frac{t_{0} c}{l_{0}}, \quad \bar{\omega}=t_{0} \omega, \quad \bar{\chi}_{3}=E_{0}^{2} \chi_{3}, \\
\bar{\varepsilon}(t) & =\frac{1}{2 \pi} \int_{0}^{\infty} \bar{\varepsilon}(\bar{\omega}) e^{-i \bar{\omega} \bar{t}} d \bar{\omega} .
\end{aligned}
$$

Далее мы будем предполагать, что для импульсов длительностью менее одного полного цикла можно обеспечить локализацию спектра импульса вблизи частоты $\bar{\omega}=0[6]$. С учетом этого выполним последовательно преобразование Фурье интегрального члена в правой части уравнения (2) по временно́й координате, разложение Тейлора для $\bar{k}(\bar{\omega})$ вблизи $\bar{\omega}=0$ и обратное преобразование Фурье. В результате получим следующее безразмерное волновое уравнение для импульса, распространяющегося в $z$-направлении:

$$
\left(\bar{\nabla}_{\perp}^{2}+\frac{\partial^{2}}{\partial \bar{z}^{2}}\right) \bar{E}\left(\bar{r}_{\perp}, \bar{z}, \bar{t}\right)=-\left[\sum_{n=0}^{\infty} \frac{1}{n !}\left(\frac{\partial^{n} \bar{k}}{\partial \bar{\omega}^{n}}\right)_{\bar{\omega}=0}\left(i \frac{\partial}{\partial \bar{t}}\right)^{n}\right]^{2} \bar{E}\left(\bar{r}_{\perp}, \bar{z}, \bar{t}\right)+
$$




$$
+\frac{\bar{\chi}_{3}}{\bar{c}^{2}} \frac{\partial^{2}}{\partial \bar{t}^{2}}\left(\left[\bar{E}\left(\bar{r}_{\perp}, \bar{z}, \bar{t}\right) \cdot \bar{E}\left(\bar{r}_{\perp}, \bar{z}, \bar{t}\right)\right] \bar{E}\left(\bar{r}_{\perp}, \bar{z}, \bar{t}\right)\right)
$$

где $\bar{k}=l_{0} k, \bar{x}=x / l_{0}, \bar{y}=y / l_{0}, \bar{z}=z / l_{0}$ и $\bar{\nabla}_{\perp}^{2}=\partial^{2} / \partial \bar{x}^{2}+\partial^{2} / \partial \bar{y}^{2}$.

Чтобы самосогласованным образом учесть эффекты дисперсии, дифракции и диссипации в зависимости от физической ситуации в каждой задаче, введем новые переменные $R_{\perp}=\epsilon^{a} \bar{r}_{\perp}, \bar{z}^{\prime}=\epsilon \bar{z}, \bar{T}=\epsilon \bar{t}$, где $a$ - число, $\epsilon \ll 1-$ малый (скейлинговый) параметр, а $R_{\perp}=\left(\epsilon^{a} x / l_{0}, \epsilon^{a} y / l_{0}\right)$. Как мы увидим ниже, эта процедура позволяет различать режимы распространения импульсов с различными относительными величинами дисперсии, дифракции, диссипации и нелинейности в терминах степеней малого параметра $\epsilon$. В результате получаем

$$
\begin{aligned}
& \left(\epsilon^{2 a} \bar{\nabla}_{\perp}^{2}+\epsilon^{2} \frac{\partial^{2}}{\partial z^{\prime 2}}\right) \bar{E}\left(\bar{R}_{\perp}, \bar{z}^{\prime}, \bar{T}\right)=-\left[\sum_{n=0}^{\infty} \frac{1}{n !}\left(\frac{\partial^{n} \bar{k}}{\partial \bar{\omega}^{n}}\right)_{\bar{\omega}=0}\left(i \epsilon \frac{\partial}{\partial \bar{T}}\right)^{n}\right]^{2} \bar{E}\left(\bar{R}_{\perp}, \bar{z}^{\prime}, \bar{T}\right)+ \\
& +\epsilon^{2} \frac{\bar{\chi}_{3}}{\bar{c}^{2}} \frac{\partial^{2}}{\partial \bar{T}^{2}}\left(\left[\bar{E}\left(\bar{R}_{\perp}, \bar{z}^{\prime}, \bar{T}\right) \cdot \bar{E}\left(\bar{R}_{\perp}, \bar{z}^{\prime}, \bar{T}\right)\right] \bar{E}\left(\bar{R}_{\perp}, \bar{z}^{\prime}, \bar{T}\right)\right)
\end{aligned}
$$

Теперь, поскольку справедливо условие, что амплитуда электрического поля $\bar{E}$ в приведенном выше волновом уравнении вещественна, заключаем, что $\operatorname{Re} \bar{\varepsilon}$ является четной функцией $\bar{\omega}$, a $\operatorname{Im} \bar{\varepsilon}-$ нечетной функцией $\bar{\omega}$. Выделим в комплексном волновом числе $\bar{k}$ вещественную и мнимую части:

$$
\begin{aligned}
\bar{k} & =\bar{\beta}(\bar{\omega})+\frac{i}{2} \bar{\alpha}(\bar{\omega}), \quad \bar{\beta}(\bar{\omega})=\operatorname{Re}\left(\frac{\bar{\omega}}{\bar{c}} \bar{\varepsilon}\right)=\frac{\bar{\omega}}{\bar{c}} \sqrt{|\bar{\varepsilon}|} \cos \frac{\phi}{2}, \\
\bar{\alpha}(\bar{\omega}) & =\operatorname{Im}\left(\frac{\bar{\omega}}{\bar{c}} \bar{\varepsilon}\right)=\frac{\bar{\omega}}{\bar{c}} \sqrt{|\bar{\varepsilon}|} \sin \frac{\phi}{2}, \quad \frac{\phi}{2}=\operatorname{tg}^{-1}\left(\frac{\operatorname{Im} \bar{\varepsilon}}{\operatorname{Re} \bar{\varepsilon}}\right) .
\end{aligned}
$$

Заметим, что $\phi(\bar{\omega})$ является нечетной функцией $\bar{\omega}$. Тогда заключаем, что $\bar{\beta}(\bar{\omega})-$ нечетная функция $\bar{\omega}$, а $\bar{\alpha}(\bar{\omega})$ - четная функция $\bar{\omega}$. Поэтому производные четного порядка функции $\bar{\beta}(\bar{\omega})$ по $\bar{\omega}$ обращаются в нуль, а также обращаются в нуль производные нечетного порядка функции $\bar{\alpha}(\bar{\omega})$ по $\bar{\omega}$ :

$$
\bar{\beta}_{0}^{(2 m)} \equiv\left(\frac{d^{2 m} \bar{\beta}}{d \bar{\omega}^{2 m}}\right)_{\bar{\omega}=0}=0, \quad \bar{\alpha}_{0}^{(2 m+1)} \equiv\left(\frac{d^{2 m+1} \bar{\alpha}}{d \bar{\omega}^{2 m+1}}\right)_{\bar{\omega}=0}=0
$$

при $m=0,1,2, \ldots$ и $\bar{\beta}(\bar{\omega})_{\omega=0}=0, \bar{\alpha}(\bar{\omega})_{\omega=0}=0$. В результате имеем

$$
-\left[\sum_{n=0}^{\infty} \frac{1}{n !}\left(\frac{\partial^{n} \bar{k}}{\partial \bar{\omega}^{n}}\right)_{\bar{\omega}=0}\left(i \epsilon \frac{\partial}{\partial \bar{T}}\right)^{n}\right]^{2}=-\left(-\epsilon^{2}\left(\bar{\beta}_{0}^{(1)}\right)^{2} \frac{\partial^{2}}{\partial \bar{T}^{2}}+2 i \epsilon \bar{\beta}_{0}^{(1)} \widehat{D}\left(\partial_{\bar{T}}\right) \frac{\partial}{\partial \bar{T}}+\widehat{D}^{2}\left(\partial_{\bar{T}}\right)\right),
$$

где оператор $\widehat{D}$ имеет вид

$$
\widehat{D}\left(\partial_{\bar{T}}\right)=\sum_{m=0}^{\infty} i^{2 m+3}\left[\epsilon^{2 m+3} \frac{\bar{\beta}_{0}^{(2 m+3)}}{(2 m+3) !}\left(\frac{\partial}{\partial \bar{T}}\right)^{2 m+3}+\epsilon^{2 m+2} \frac{\bar{\alpha}_{0}^{(2 m+2)}}{2(2 m+2) !}\left(\frac{\partial}{\partial \bar{T}}\right)^{2 m+2}\right] .
$$

Перейдем теперь к движущейся системе отсчета, вводя для этого новые переменные [7] $\tau=\bar{T}-\bar{\beta}_{0}^{(1)} z^{\prime}=\bar{T}-z^{\prime} / v_{\mathrm{g}}, \xi=\epsilon^{\gamma} z^{\prime}$, где $v_{\mathrm{g}}-$ групповая скорость (которая 
тождественна фазовой скорости при $\bar{\omega}=0)$, а $\gamma$ - константа, и получим

$$
\begin{aligned}
& \epsilon^{2 a} \bar{\nabla}_{\bar{R}_{\perp}}^{2} \bar{E}\left(\bar{R}_{\perp}, \xi, \tau\right)-2 \bar{\beta}_{0}^{(1)} \epsilon^{2+\gamma} \frac{\partial^{2}}{\partial \tau \partial \xi} \bar{E}\left(\bar{R}_{\perp}, \xi, \tau\right)+\epsilon^{2+2 \gamma} \frac{\partial^{2}}{\partial \xi^{2}} \bar{E}\left(\bar{R}_{\perp}, \xi, \tau\right)+ \\
& +2 i \epsilon^{3} \bar{\beta}_{0}^{(1)} \partial_{\tau} \overline{\widehat{D}}\left(\partial_{\tau}\right) \bar{E}\left(\bar{R}_{\perp}, \xi, \tau\right)-\epsilon^{4} \overline{\widehat{D}}^{2} \bar{E}\left(\bar{R}_{\perp}, \xi, \tau\right)= \\
& =\epsilon^{2} \frac{\bar{\chi}_{3}}{\bar{c}^{2}} \frac{\partial^{2}}{\partial \tau^{2}}\left[\bar{E}\left(\bar{R}_{\perp}, \xi, \tau\right) \cdot \bar{E}\left(\bar{R}_{\perp}, \xi, \tau\right) \bar{E}\left(\bar{R}_{\perp}, \xi, \tau\right)\right]
\end{aligned}
$$

где оператор $\widehat{\widehat{D}}$ имеет вид

$$
\overline{\widehat{D}}\left(\partial_{\tau}\right)=\sum_{m=0}^{\infty} i^{2 m+3}\left[\epsilon^{2 m+1} \frac{\bar{\beta}_{0}^{(2 m+3)}}{(2 m+3) !}\left(\frac{\partial}{\partial \tau}\right)^{2 m+3}+\epsilon^{2 m} \frac{\bar{\alpha}_{0}^{(2 m+2)}}{2(2 m+2) !}\left(\frac{\partial}{\partial \tau}\right)^{2 m+2}\right]
$$

Рассмотрим случай, когда дифракция и дисперсия сравнимы в главном порядке по $\epsilon$ при $m=0$. Это дает $a=\gamma=2$. Далее, для слабой нелинейности в слабо диссипативной среде будем считать, что эффект диссипации имеет порядок $\epsilon$, а нелинейные эффекты имеют порядок $\epsilon^{2}$. Тогда масштабное преобразование соответствующих коэффициентов $\bar{\alpha}_{0}^{(2)}=\epsilon \bar{\alpha}_{0}^{(2)}, \bar{\chi}_{3}=\epsilon^{2} \bar{\chi}_{3}$ дает эволюционное уравнение

$$
\bar{\nabla}_{\perp}^{2} \bar{E}-2 \bar{\beta}_{0}^{(1)}\left(\frac{\partial^{2} \bar{E}}{\partial \tau \partial \xi}-\frac{\bar{\beta}_{0}^{(3)}}{6} \frac{\partial^{4} \bar{E}}{\partial \tau^{4}}-\frac{\bar{\alpha}_{0}^{(2)}}{4} \frac{\partial^{3} \bar{E}}{\partial \tau^{3}}\right)=\frac{\bar{\chi}_{3}}{\bar{c}^{2}} \frac{\partial^{2}}{\partial \tau^{2}}\left(\bar{E}^{2} \bar{E}\right) .
$$

Приведенное дифференциальное уравнение в частных производных описывает пространственно-временную эволюцию электромагнитных импульсов длительностью в несколько циклов и менее одного полного цикла в керровской нелинейной среде с дисперсией, диссипацией и дифракцией. Ясно, что эффект дисперсии возникает из-за присутствия производной четвертого порядка электрического поля с коэффициентом, являющимся производной третьего порядка вещественной части $k$ по $\omega$. Эффект диссипации обусловлен наличием в уравнении производной второго порядка электрического поля с коэффициентом, являющимся производной второго порядка $k$ по $\omega$. Заметим, что мы не налагали никаких ограничений на закон дисперсии в рассматриваемой среде (задаваемый как $\bar{\varepsilon}(\bar{\omega})$, или что эквивалентно, каK $\bar{\chi}(\bar{\omega}))$.

\section{2. ЧИСЛЕННЫЕ РЕЗУЛЬТАТЫ ДЛЯ ИМПУЛЬСА ДЛИТЕЛЬНОСТЬЮ ОДИН ЦИКЛ}

Теперь представим результаты численного моделирования одномерного распространения импульса длительностью один цикл с длиной волны 0.8 мкм в среде с кубичной нелинейностью. Уравнение распространения имеет вид

$$
\frac{\partial^{2} \bar{E}}{\partial \tau \partial \xi}=\frac{\bar{\beta}_{0}^{(3)}}{6} \frac{\partial^{4} \bar{E}}{\partial \tau^{4}}+\frac{\bar{\alpha}_{0}^{(2)}}{4} \frac{\partial^{3} \bar{E}}{\partial \tau^{3}}-\frac{\bar{\chi}_{3}}{2 \bar{\beta}_{0}^{(1)} \bar{c}^{2}} \frac{\partial^{2}}{\partial \tau^{2}}\left(\bar{E}^{2} \bar{E}\right)
$$

Мы проинтегрировали это уравнение численно при различных значениях параметра $\bar{\chi}_{3}$. Результаты приведены для значения $\bar{\chi}_{3}=0.05$. Использовались следующие физические параметры: $t_{0}=10^{-14} \mathrm{c}, \bar{\beta}_{0}^{(3)}=0.0041125$. Это соответствует дисперсионному коэффициенту третьего порядка $\beta^{(3)}=0.0274$ фc $^{3} /$ мкм [8], $\bar{\alpha}_{0}^{(2)}=0.05391$, 


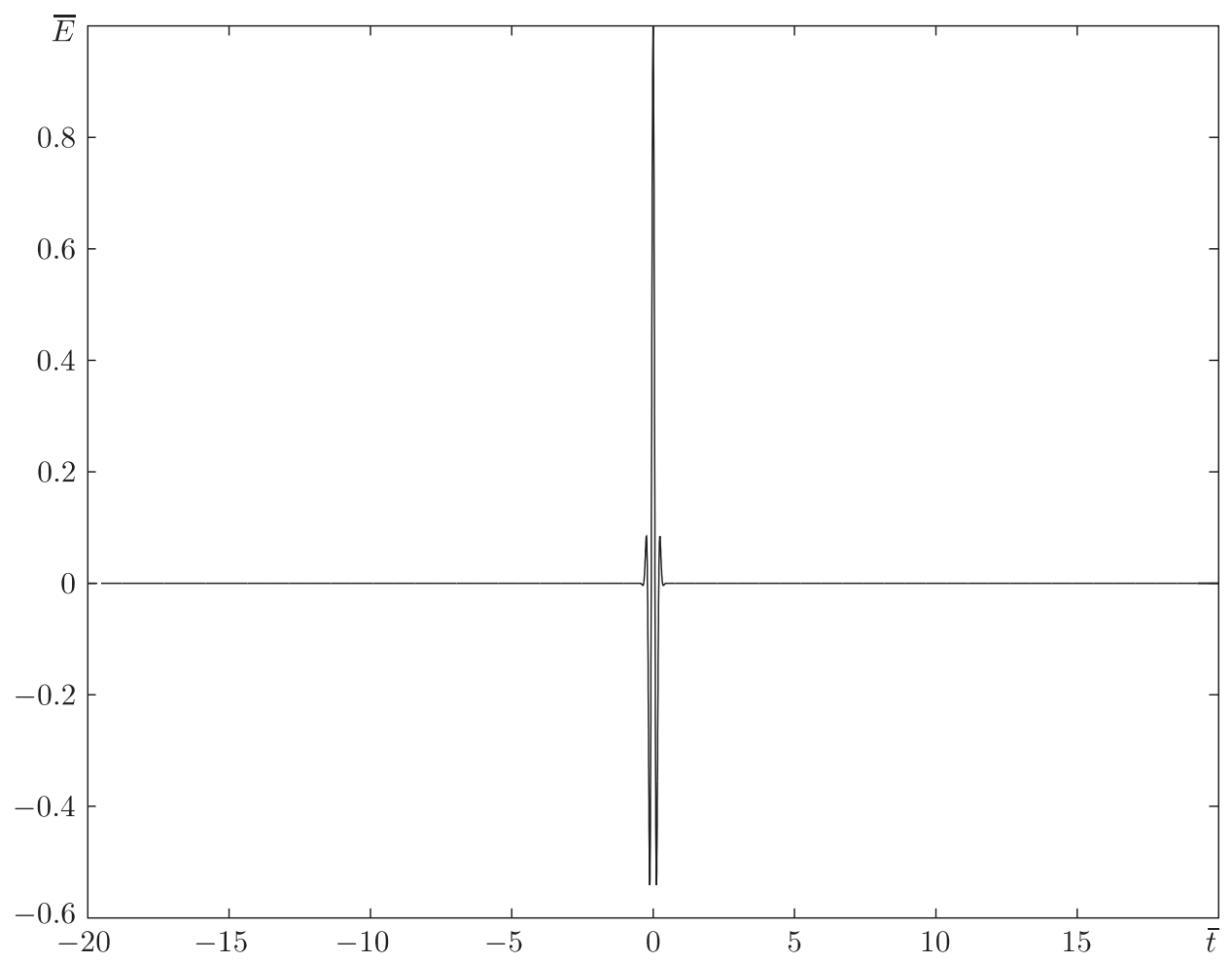

Рис. 1. Безразмерное электрическое поле $\bar{E}$ импульса длительностью один цикл при $\lambda=0.8$ мкм как функция безразмерного времени $\bar{t}$.

т.е. дисперсионному коэффициенту второго порядка $\beta^{(2)}=0.03592 \mathrm{\phi c}^{2} / \mathrm{мкм} \mathrm{[8]}$ и $\chi^{(3)}=2.234 \cdot 10^{-22} \mathrm{~m}^{2} / \mathrm{B}^{2}$, что соответствует $n_{2}=0.6052 \cdot 10^{-22} \mathrm{~m}^{2} / \mathrm{B}^{2}$. Характерное электрическое поле $E_{0}$, использованное выше для получения безразмерной величины, взято равным $E_{0}=1.46 \cdot 10^{10.5} \mathrm{~B} /$ м. При заданном значении дисперсионного коэффициента третьего порядка характерная длина дисперсии для импульса с полной шириной на половине максимума, равной 2.67 фс, что соответствует импульсу длительностью один цикл с длиной волны 0.8 мкм, оказывается равной $l_{\mathrm{d}} \approx 150.0 \cdot 10^{-6} \mathrm{м}$, что мы и принимаем за характерную длину $l_{0}$, используемую при определении безразмерной величины. Далее, если вычислить характерное расстояние самопроизвольного увеличения крутизны как $z_{\mathrm{s}}=\sqrt{e / 2} L_{\mathrm{nl}} \omega_{0} T_{0}$ [8], где $L_{\mathrm{nl}}$ - характерная длина нелинейности, то для заданных параметров волокна оно получается равным $z_{\mathrm{s}} \approx 14.45$ мкм.

Безразмерное электрическое поле входного импульса, соответствующее импульсу длительностью один цикл с длиной волны 0.8 мкм, имеет вид

$$
\bar{E}(\tau)=\exp \left(\left(-\frac{\tau}{\tau_{\mathrm{p}} / 1.67}\right)^{2}\right) \cos \left(\bar{\omega}_{0} \tau\right),
$$

где $\tau_{\mathrm{p}}$ - полная ширина на половине максимума. Это электрическое поле показано на рис. 1. 


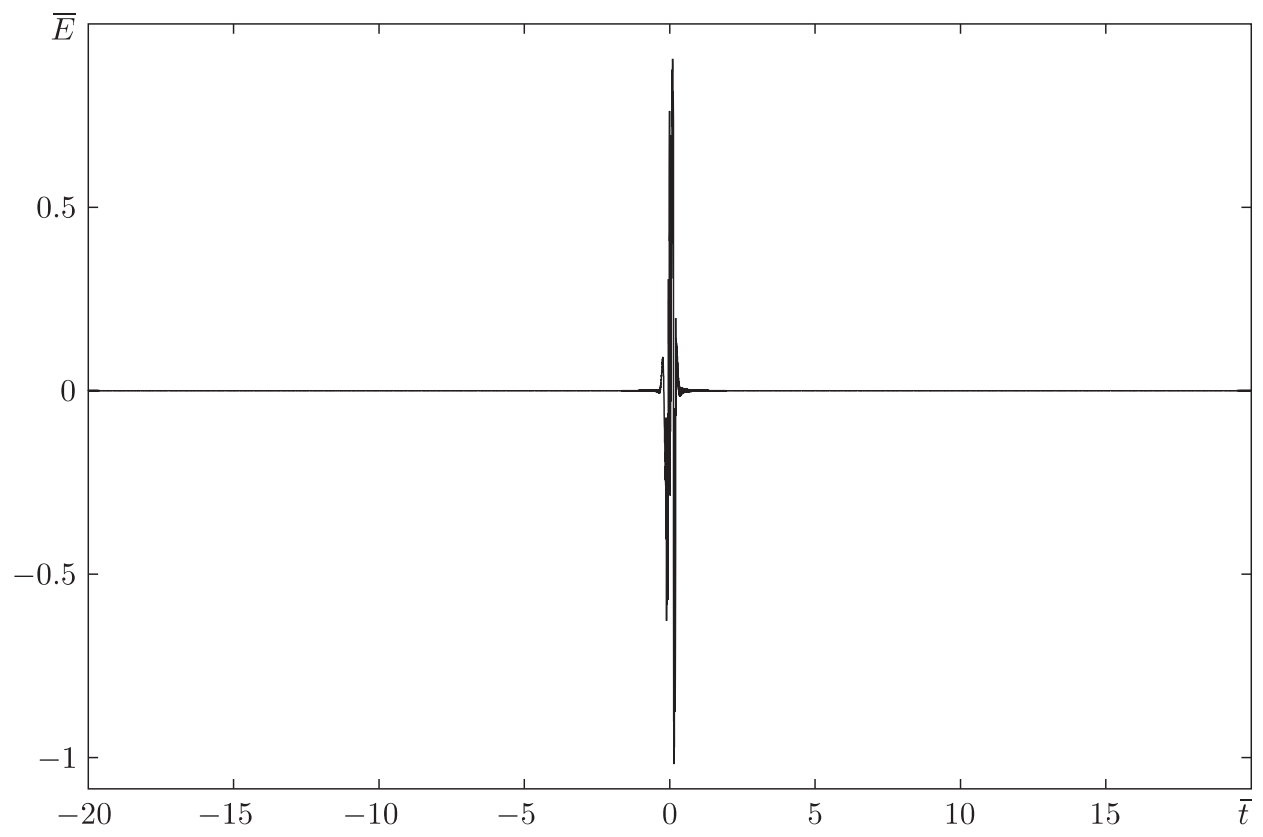

Рис. 2. Безразмерное электрическое поле $\bar{E}$ импульса длительностью один цикл при $\lambda=0.8$ мкм как функция безразмерного времени $\bar{t}$ после прохождения 13.5 мкм при учете только нелинейности.

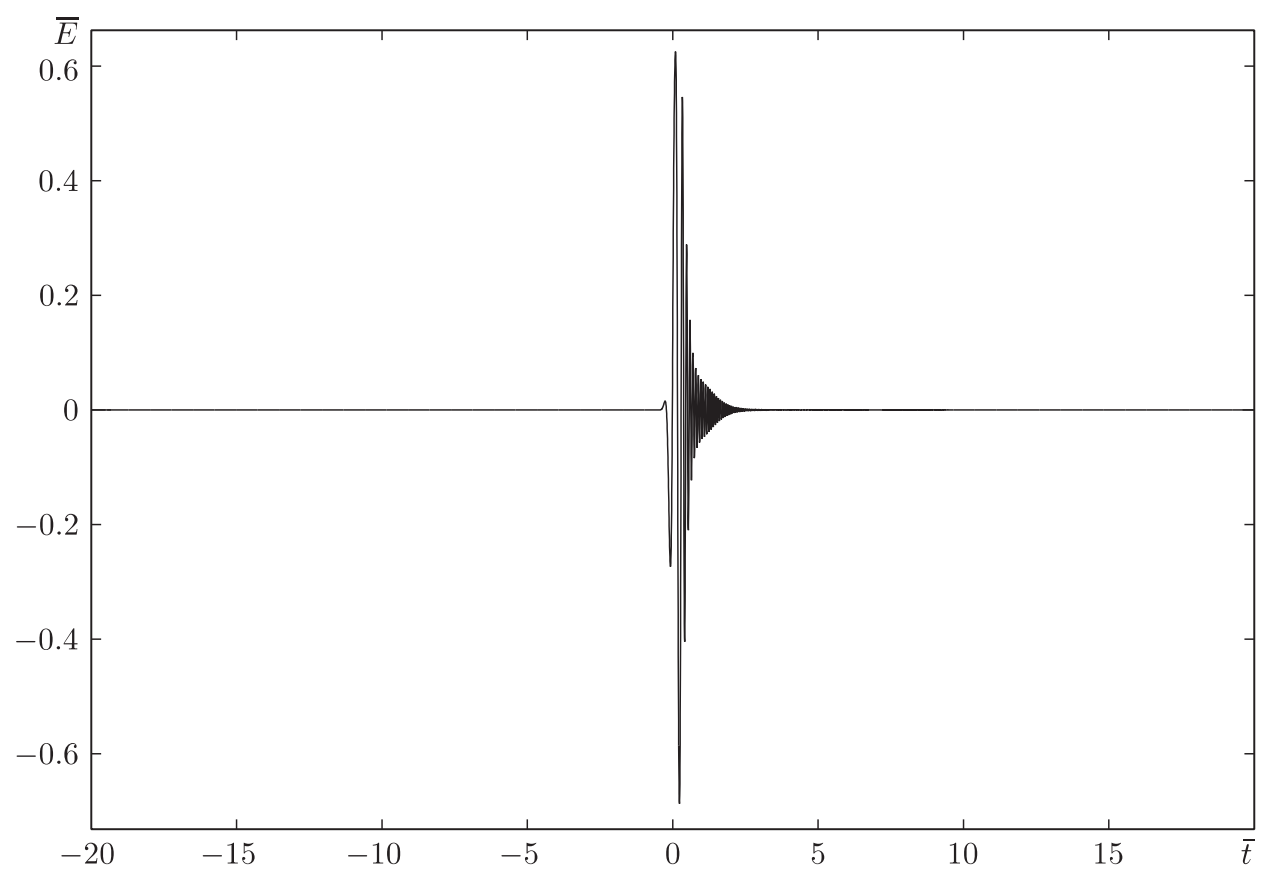

Рис. 3. То же, что и на рис. 2, но при учете и нелинейности, и дисперсии. 


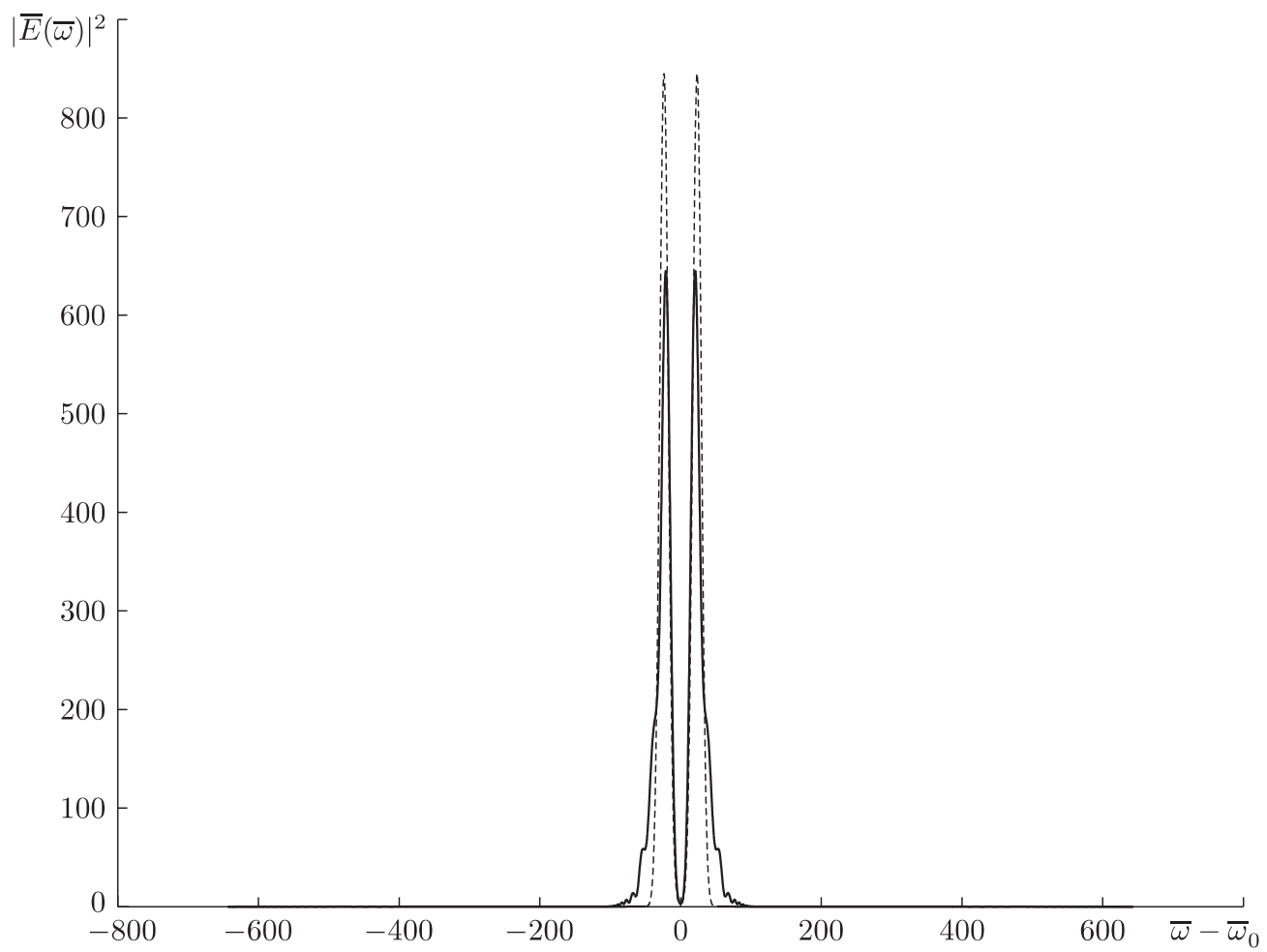

Рис. 4. Безразмерная интенсивность $|\bar{E}(\bar{\omega})|^{2}$ импульса длительностью один цикл при $\lambda=0.8$ мкм после прохождения 13.5 мкм как функция безразмерной частоты $\bar{\omega}-\bar{\omega}_{0}$ при учете и нелинейности, и дисперсии.

На рис. 2 и 3 показано безразмерное электрическое поле $\bar{E}$ импульса как функция безразмерного времени $\bar{t}$ после прохождения импульсом расстояния 13.5 мкм, что близко к характерной длине самопроизвольного увеличения крутизны $\left(z_{\mathrm{s}} \approx\right.$ 14.45 мкм). Рис. 2 соответствует случаю, когда учитывается только нелинейность, а рис. 3 - случаю, когда присутствуют и нелинейность, и дисперсия.

Как видно, в обоих случаях импульс претерпевает самопроизвольное увеличение крутизны, из-за которого центр импульса сдвигается по направлению к заднему фронту. Эффект дисперсии проявляется на рис. 3 в генерируемых осцилляторных структурах. Эти осцилляторные структуры оказываются у заднего фронта плотнее, чем у переднего. Данная асимметрия обусловлена эффектом самопроизвольного увеличения крутизны. В отсутствие самопроизвольного увеличения крутизны эта осцилляторная структура должна была бы быть симметричной, поскольку дисперсия связана с производной четвертого порядка электрического поля [9]. На рис. 4 представлен спектр безразмерной интенсивности импульса после прохождения 13.5 мкм. Штриховая кривая соответствует входному импульсу, сплошная выходному. Ясно видно, что нелинейность порождает новые частоты, из-за чего выходной импульс уширяется. На рис. 5 показано безразмерное электрическое поле 


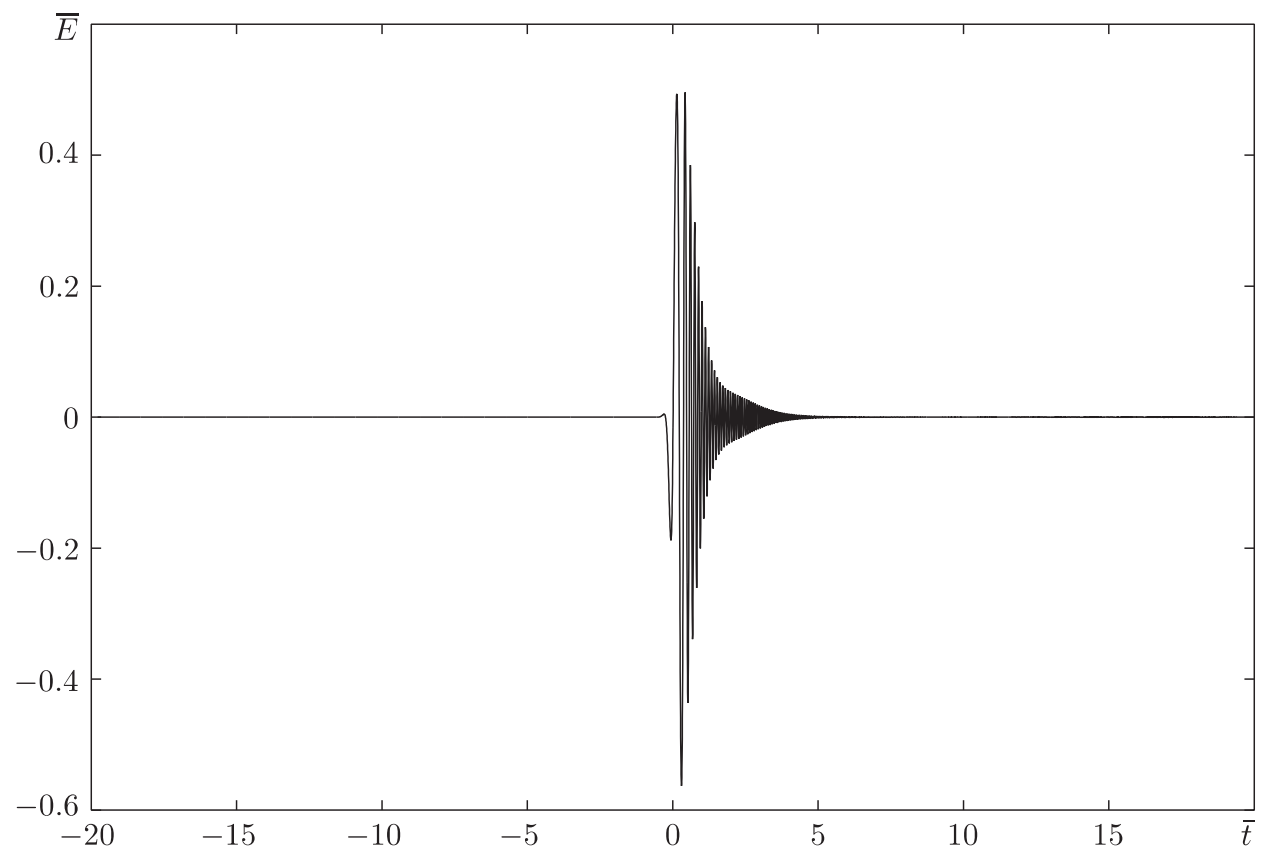

Рис. 5. Безразмерное электрическое поле $\bar{E}$ импульса длительностью один цикл при $\lambda=0.8$ мкм как функция безразмерного времени $\bar{t}$ после прохождения 27.0 мкм при учете нелинейности и дисперсии.

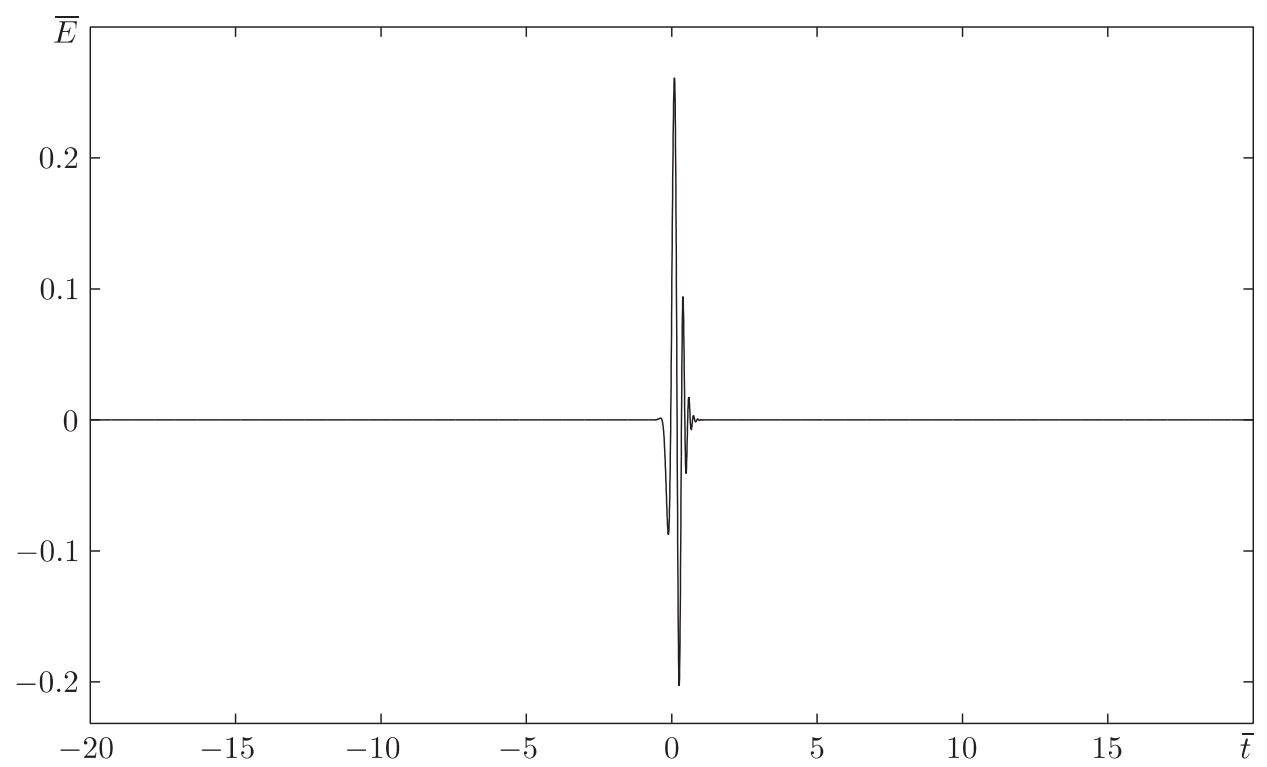

Рис. 6. То же, что и на рис. 5, но при учете нелинейности, дисперсии и диссипации. 
импульса после прохождения 27.0 мкм в случае, когда учитываются и нелинейность, и дисперсия. Характер распространения импульса остается в целом тем же, за исключением того, что порождается все больше и больше осцилляторных структур. Амплитуда пика убывает вследствие того, что ширина импульса возрастает под действием доминирующих эффектов дисперсии.

Наконец, на рис. 6 приведено электрическое поле импульса после прохождения 13.5 мкм, когда вместе с нелинейностью и дисперсией в нашем численном решении учтен также член, описывающий затухание. Как видно, затухание приводит к значительному уменьшению амплитуды пика электрического поля импульса. Отметим еще уменьшение числа осцилляторных структур на краях, что может быть вызвано редукцией эффекта самопроизвольного возрастания крутизны при уменьшении интенсивности электрического поля.

\section{3. ЗАКЛЮЧЕНИЕ}

Мы привели нелинейное уравнение эволюции для импульсов длительностью в несколько циклов и менее одного полного цикла, которое было выведено из нелинейного волнового уравнения для вещественного электрического поля импульса. Новизна предложенного уравнения состоит в том, что в главном порядке эффект дисперсии обусловлен наличием производной четвертого порядка электрического поля с коэффициентом, являющимся производной третьего порядка вещественной части $k$ по $\omega$. Эффект диссипации возникает благодаря производной второго порядка электрического поля с коэффициентом, являющимся производной второго порядка $k$ по $\omega$. Кроме того, мы выполнили численные расчеты для одномерного распространения импульса длительностью один цикл с длиной волны 0.8 мкм в среде с кубичной нелинейностью. Приведенное обсуждение полученных результатов позволяет заключить, что представленное модельное уравнение действительно описывает основные свойства распространения импульсов, обусловленные нелинейностью, дисперсией и диссипацией, а также показывает, что дисперсия и диссипация играют роль стабилизирующего эффекта, предохраняющего от самопроизвольного увеличения крутизны и развала импульса вблизи и после характерной длины самопроизвольного увеличения крутизны.

Благодарности. Я благодарен доктору Дж. Биндиону за его замечание в ходе моей лекции, которое помогло исправить ошибку в численном анализе и избавиться от ложного усиления пиковой амплитуды импульса. Я сердечно благодарен профессору Дж. П. Агравалу за плодотворные дискуссии во время его пребывания у нас на факультете. Я также благодарю В. Мишру за помощь в численных расчетах.

\section{Список литературы}

[1] J. H. B. Nijhof, H. A. Ferverda, B. J. Hoenders, Pure Appl. Opt., 4:3 (1995), 199-218.

[2] T. Brabec, F. Krausz, Phys. Rev. Lett., 78:17 (1997), 3282-3285.

[3] V. G. Bespalov, S. A. Kozlov, Yu. A. Shpolyanskiy, I. A. Walmsley, Phys. Rev. A, 66:1 (2002), 013811.

[4] T. Schäfer, C. E. Wayne, Physica D, 196:1-2 (2004), 90-105.

[5] T. Tsurumi, J. Phys. Soc. Japan, 75:2 (2006), 024002. 
[6] M. B. Johnston, D. M. Whittaker, A. Dowd, A. G. Devies, E. H. Linfield, X. Li, D. A. Ritchie, Opt. Lett., 27:21 (2002), 1935-1937.

[7] T. Taniuti, C.-C. Wei, J. Phys. Soc. Japan, 24:4 (1968), 941-946.

[8] S. Diddams, J.-C. Diels, J. Opt. Soc. Amer. B, 13:6 (1996), 1120-1129.

[9] M. C. Stefanovic, A. S. Panajotovic, D. M. Milovic, "Pulse deformation due to fourth order dispersion and inband interference", Proc. 8th Internat. Conf. Telecomm. Modern Satellite, Cable and Broadcasting Serv., TELSIKS, 2007 (September 26-28, 2007, Niš, Serbia), Univ. Niš, Serbia, 2007, 609-612. 